\title{
Calculation of the coherent synchrotron radiation impedance from a wiggler
}

\author{
Juhao Wu, ${ }^{*}$ Tor O. Raubenheimer, ${ }^{\dagger}$ and Gennady V. Stupakov ${ }^{\ddagger}$ \\ Stanford Linear Accelerator Center, Stanford University, Stanford, California 94309
}

(Received 22 January 2003; published 9 April 2003)

\begin{abstract}
Most studies of coherent synchrotron radiation (CSR) have considered only the radiation from independent dipole magnets. However, in the damping rings of future linear colliders, a large fraction of the radiation power will be emitted in damping wigglers. In this paper, the longitudinal wakefield and impedance due to CSR in a wiggler are derived in the limit of a large wiggler parameter $K$. After an appropriate scaling, the results can be expressed in terms of universal functions, which are independent of $K$. Analytical asymptotic results are obtained for the wakefield in the limit of large and small distances, and for the impedance in the limit of small and high frequencies.
\end{abstract}

DOI: 10.1103/PhysRevSTAB.6.040701

\section{INTRODUCTION}

Many modern advanced accelerator projects [1-3] call for short bunches with low emittance and high peak current where coherent synchrotron radiation (CSR) effects may play an important role. CSR is emitted at wavelengths longer than or comparable to the bunch length whenever the beam is deflected $[4,5]$. The stringent beam requirements needed for short wavelength selfamplified spontaneous emission free-electron lasers have led to intensive theoretical and experimental studies [6-17] over the past few years where the focus has been on the magnetic bunch compressors required to obtain the high peak currents. In addition to these single-pass cases, it is also possible that CSR might cause a microwavelike beam instability in storage rings. A theory of such an instability in a storage ring has been recently proposed in Ref. [18] with experimental evidence published in [19]. Other experimental observations [20-24] may also be associated with a CSR-driven instability as supported by additional theoretical studies [25-27].

The previous study of the CSR induced instability assumed that the impedance is generated by the synchrotron radiation of the beam in the storage ring bending magnets [18]. In some cases [e.g., the Next Linear Collider (NLC) damping ring [28]], a ring will include magnetic wigglers which introduce an additional contribution to the radiation impedance. The analysis of the microwave instability in such a ring requires knowledge of the impedance of the synchrotron radiation in the wiggler. Although there have been earlier studies of the coherent radiation from a wiggler or undulator $[29,30]$, the results
PACS numbers: 29.27.Bd, 41.60.Ap, 41.60.Cr, 41.75.Fr

of these papers cannot be used directly for the stability analysis.

In this paper, we derive the CSR wake and impedance for a wiggler. We focus our attention on the limit of a large wiggler parameter $K$ because this is the most interesting case for practical applications. It also turns out that, in this limit, the results can be expressed in terms of universal functions of a single variable after an appropriate normalization.

The paper is organized as follows. In Sec. II, we write down equations for the energy loss of a beam in a wiggler. We then derive the synchrotron radiation wakefield in the limit of a large wiggler parameter $K$ in Sec. III. In Sec. IV, we obtain the synchrotron radiation impedance for a wiggler, and in Sec. V we discuss our results.

\section{ENERGY LOSS AND LONGITUDINAL WAKE IN WIGGLER}

The longitudinal wake is directly related to the rate of energy loss $d \mathcal{E} / d t$ of an electron in the beam propagating in a wiggler. For a planar wiggler, a general expression for $d \mathcal{E} / d t$ as a function of the position $s$ of the electron in the bunch and the coordinate $z$ in the wiggler was derived in Ref. [30]. We reproduce here the results of that work using the authors' notation:

$$
\frac{d \mathcal{E}}{c d t}=e^{2} k_{w} \int_{-\infty}^{s} d s^{\prime} D\left(\hat{s}-\hat{s}^{\prime}, K, \hat{z}\right) \frac{d \lambda\left(s^{\prime}\right)}{d s^{\prime}}
$$

where $\lambda(s)$ is the bunch linear density,

$$
D(\hat{s}, K, \hat{z})=\frac{1}{\hat{s}}-2 \frac{\Delta-K^{2} B(\Delta, \hat{z})[\sin \Delta \cos \hat{z}+(1-\cos \Delta) \sin \hat{z}]}{\Delta^{2}+K^{2} B^{2}(\Delta, \hat{z})},
$$

*Electronic address: jhwu@SLAC.Stanford.EDU

"Electronic address: tor@SLAC.Stanford.EDU

"Electronic address: stupakov@SLAC.Stanford.EDU

$$
\begin{aligned}
B(\Delta, \hat{z})= & (1-\cos \Delta-\Delta \sin \Delta) \cos \hat{z} \\
& +(\Delta \cos \Delta-\sin \Delta) \sin \hat{z},
\end{aligned}
$$

and $\Delta$ is the solution of the transcendental equation 


$$
\begin{aligned}
\hat{s}=\frac{\Delta}{2}\left(1+\frac{K^{2}}{2}\right)+\frac{K^{2}}{4 \Delta}\{ & {[2(1-\cos \Delta)-\Delta \sin \Delta] } \\
& \times(\cos \Delta \cos 2 \hat{z}+\sin \Delta \sin 2 \hat{z}) \\
& -2(1-\cos \Delta)\} .
\end{aligned}
$$

In the above equations, we use the following dimensionless variables: $\hat{s}=\gamma^{2} k_{w} s$ and $\hat{z}=k_{w} z$. The parameter $\Delta$ is equal to $k_{w}\left(z-z_{r}\right)$, where $z$ and $z_{r}$ are the projected coordinates on the wiggler axis of the current position of the test particle and the retarded position of the source particle, respectively. The internal coordinate $s$ is defined so that the bunch head corresponds to a larger value of $s$ than the tail. The wiggler parameter $K$ is approximately $K \approx 93.4 B_{w} \lambda_{w}$, with $B_{w}$ the peak magnetic field of the wiggler in units of Tesla and $\lambda_{w}$ the period in meters. In addition, $\gamma$ is the Lorentz factor, $e$ is the electron charge, $c$ is the speed of light in vacuum, and $k_{w}=2 \pi / \lambda_{w}$ is the wiggler wave number. Note that the function $D$ is a periodic function of $\hat{z}$ with a period equal to $\pi$. Also note that, despite assuming $K \gg 1$, we still assume a small-angle orbit approximation, i.e., $K / \gamma \ll 1$.

We introduce the longitudinal wake $W(s)$ of the bunch as the rate of the energy change averaged over the $z$ coordinate:

$$
W(s)=-\frac{1}{e^{2}} \frac{d \overline{\mathcal{E}}}{c d t}=-k_{w} \int_{-\infty}^{s} d s^{\prime} G\left(s-s^{\prime}\right) \frac{d \lambda\left(s^{\prime}\right)}{d s^{\prime}},
$$

where

$$
G(s)=\frac{1}{\pi} \int_{0}^{\pi} d \hat{z} D(\hat{s}, K, \hat{z}),
$$

and we dropped $K$ from the list of arguments of the function $G$. The positive values of $W$ correspond to the energy loss and the negative values imply the energy gain. The usual longitudinal wake $w(s)$ corresponding to the interaction of two particles is then defined as

$$
w(s)=-k_{w} \frac{d G(s)}{d s},
$$

so that

$$
W(s)=\int_{-\infty}^{s} d s^{\prime} w\left(s-s^{\prime}\right) \lambda\left(s^{\prime}\right)
$$

Note that the wake Eq. (7) is localized in front of the particle and vanishes behind it; $w=0$ for $s<0$.

In the limit of large $K$, we can neglect unity in the first bracket of Eq. (4), assuming that $K^{2} / 2 \gg 1$. Such an approximation is valid, if we are not interested in the very short distances of order of $\left(K k_{w} \gamma^{2}\right)^{-1}(0.5 \AA$ for the NLC damping ring wiggler [28]). We also introduce a new variable $\zeta \equiv \hat{s} / K^{2}$ which eliminates the parameter $K$ from Eq. (4):

$$
\begin{aligned}
\zeta(\Delta, \hat{z})=\frac{\Delta}{4}+\frac{1}{4 \Delta}\{ & {[2(1-\cos \Delta)-\Delta \sin \Delta] } \\
& \times(\cos \Delta \cos 2 \hat{z}+\sin \Delta \sin 2 \hat{z}) \\
& -2(1-\cos \Delta)\} .
\end{aligned}
$$

In this limit, the expression for $D$, Eq. (2), can also be simplified:

$$
D(\zeta, \hat{z})=2 \frac{\sin \Delta \cos \hat{z}+(1-\cos \Delta) \sin \hat{z}}{B(\Delta, \hat{z})},
$$

as long as $\Delta$ is not too small, $\Delta \gg 1 / K$. Again, the parameter $K$ is eliminated from this equation. A detailed analysis supporting this approximation can be found in Appendix A.

\section{WAKEFIELD}

Using Eqs. (6) and (10) we find

$$
G(\zeta)=\frac{2}{\pi} \int_{0}^{\pi} d \hat{z} \frac{\sin \Delta \cos \hat{z}+(1-\cos \Delta) \sin \hat{z}}{B(\Delta, \hat{z})}
$$

where $\Delta=\Delta(\zeta, \hat{z})$ is implicitly determined by Eq. (9). The integrand in this equation has singularities at points $\hat{z}=\hat{z}_{s}$ where $B\left(\Delta\left(\zeta, \hat{z}_{s}\right), \hat{z}_{s}\right)=0$. It is shown in Appendix $\mathrm{B}$ that in the vicinity of a singular point $B(\Delta(\zeta, \hat{z})) \propto\left(\hat{z}-\hat{z}_{s}\right)^{1 / 3}$, and the singularity is integrable.

We plot in Fig. 1 the function $G(\zeta)$ calculated by numerical integration. A characteristic feature of the function $G$ is the presence of cusp points, at which the function reaches local maxima and minima.

An approximate location of these cusp points and the value of the function $G$ at these points can be understood with a simple physical argument presented in Appendix C. It turns out that the minima are located at distances $s$ between the particles equal to the integer number of the fundamental radiation wavelength in the wiggler, and the maxima approximately correspond to the distance equal to an odd number of half-wavelengths. A simple analytical calculation in Appendix $\mathrm{C}$ gives the following results:

$$
G(\zeta)= \begin{cases}0 ; & \text { for } \zeta=\frac{n \pi}{2} \text { with } n=1,2, \ldots \\ -\frac{4(2 n+1) \pi}{4+[(2 n+1) \pi]^{2}} ; & \text { for } \zeta \approx \frac{(2 n+1) \pi}{4}-\frac{1}{(2 n+1) \pi} \text { with } n=0,1, \ldots\end{cases}
$$

These are the " $X$ " points in Fig. 1, showing very good agreement with the numerical result.

\section{A. Short-range limit}

In the limit $\zeta \ll 1$, it follows from Eq. (9) that $\Delta \ll 1$ as well. Equation (9) can then be solved using a Taylor expansion of the right-hand side:

$$
\Delta=\left(\frac{24 \zeta}{\cos ^{2} \hat{z}}\right)^{1 / 3}
$$

Expanding the integrand in Eq. (11), keeping only the first nonvanishing term in $\Delta$ and substituting $\Delta$ from Eq. (13) yields 


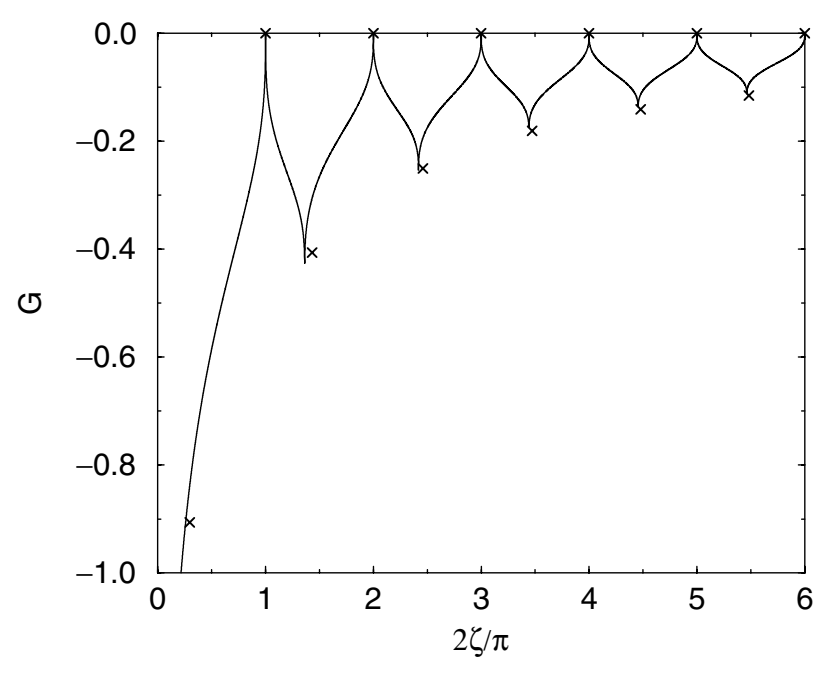

FIG. 1. The solid curve represents the $G(\zeta)$ defined in Eq. (11) as a function of the normalized coordinate $2 \zeta / \pi$. The $(\times)$ signs are the approximation given in Eq. (12).

$$
\begin{aligned}
G(\zeta) & =-\frac{1}{\pi} \frac{2}{(3 \zeta)^{1 / 3}} \int_{0}^{\pi} d \hat{z} \cos ^{2 / 3} \hat{z}=-\frac{43^{2 / 3} \Gamma\left(\frac{11}{6}\right)}{5 \sqrt{\pi} \Gamma\left(\frac{4}{3}\right)} \zeta^{-1 / 3} \\
& \approx-0.99 \zeta^{-1 / 3}
\end{aligned}
$$

The above result can also be obtained if one considers a wiggler as a sequence of bending magnets with the bending radius $R=\gamma / k_{w} K|\cos \hat{z}|$. Indeed, in a bending dipole, the corresponding $G_{\text {bend }}(s)=-2 s^{-1 / 3} /\left(3 R^{2}\right)^{1 / 3}$ $[7,8]$. Averaging $G_{\text {bend }}$ over the wiggler period yields Eq. (14). The reason why such a model gives the correct result in this limit is that the formation length of the radiation is much shorter than the wiggler period, and one can use a local approximation of the bending magnet for the wake.

\section{B. Long-range limit}

In the limit $\zeta \gg 1$, the parameter $\Delta$ is also large, and Eq. (9) can be further simplified:

$$
\zeta=\frac{\Delta}{4}-\frac{\sin \Delta \cos (\Delta-2 \hat{z})}{4}
$$

In Eq. (3), we keep only the largest term

$$
B(\Delta, \hat{z})=-\Delta \sin (\Delta-\hat{z}) .
$$

For $D$, one now finds

$$
D(\zeta, \hat{z}) \equiv \frac{F(\zeta, \hat{z})}{\zeta}
$$

with

$$
F(\zeta, \hat{z}) \equiv \frac{\sin \hat{z}}{2 \sin (\hat{z}-\Delta(\zeta, \hat{z}))}-\frac{1}{2},
$$

where the function $\Delta(\zeta, \hat{z})$ is implicitly determined by Eq. (15). Averaging over one wiggler period, we find

$$
G(\zeta) \equiv \frac{\bar{F}(\zeta)}{\zeta}
$$

with

$$
\begin{aligned}
\bar{F}(\zeta) & \equiv \frac{1}{\pi} \int_{0}^{\pi} d \hat{z} F(\zeta, \hat{z}) \\
& =\frac{1}{2 \pi}\left(-\pi+\int_{0}^{\pi} d \hat{z} \frac{\sin \hat{z}}{\sin (\hat{z}-\Delta)}\right) .
\end{aligned}
$$

It is easy to check that the function $\bar{F}$ is periodic, $\bar{F}(\zeta+\pi / 2)=\bar{F}(\zeta)$, and $\bar{F}(0)=0, \bar{F}(\pi / 4)=-1$ in agreement with Eq. (12). The average value $\bar{F}(\zeta)$ is equal to $-1 / 2$. Since $\bar{F}$ is periodic in $\zeta$ with a period of $\pi / 2$, using Eq. (20), we get a Fourier series representation for $\bar{F}(\zeta)$ :

$$
\bar{F}(\zeta)=-\frac{1}{2}+\frac{1}{2} \sum_{n=0}^{\infty}\left[J_{n}\left(\frac{2 n+1}{2}\right)-J_{n+1}\left(\frac{2 n+1}{2}\right)\right]^{2} \cos (4(2 n+1) \zeta),
$$

where $J_{n}(x)$ is the Bessel function of the first kind. Derivations of the Fourier coefficients are presented in Appendix D. In Fig. 2, we plot the function $\bar{F}(\zeta)$ defined in Eq. (21) for one period.

The corresponding long-range wake is then

$$
G(\zeta)=-\frac{1}{2 \zeta}+\frac{1}{2 \zeta} \sum_{n=0}^{\infty}\left[J_{n}\left(\frac{2 n+1}{2}\right)-J_{n+1}\left(\frac{2 n+1}{2}\right)\right]^{2} \cos (4(2 n+1) \zeta)
$$

It is worth noting that the asymptotic expression in the limit $\zeta \gg 1$ in Ref. [30] is incorrect; instead of the $\bar{F}$ function the authors obtained a sine function, which corresponds only to the fundamental mode of the radiation and neglects contributions from higher-order harmonics.

The longitudinal wake defined in Eq. (7) is plotted in Fig. 3.

\section{IMPEDANCE}

The impedance $Z(k)$ is defined as the Fourier transform of the wake,

$$
Z(k)=\int_{0}^{\infty} d s w(s) e^{-i k s}=-i k \frac{K^{2}}{\gamma^{2}} \int_{0}^{\infty} d \zeta G(\zeta) e^{-4 i\left(k / k_{0}\right) \zeta},
$$




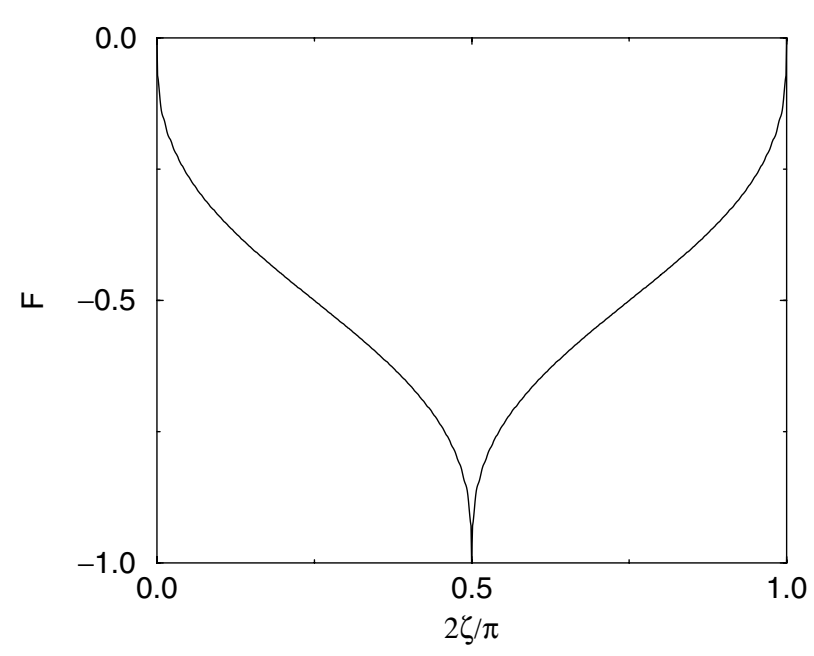

FIG. 2. Plot of $\bar{F}(\zeta)$ of Eq. (21).

where $k_{0} \equiv 4 \gamma^{2} k_{w} / K^{2}$ is the wiggler fundamental radiation wave number.

We evaluated the integral in Eq. (23) using numerically calculated values of the function $G(\zeta)$ in the interval $\left[\zeta_{\min }, \zeta_{\max }\right]$, where $\zeta_{\min } \approx 10^{-3}$ and $\zeta_{\max } \approx 50$. The contribution to the integral outside of this interval was calculated using asymptotic representations Eqs. (14) and (22).

The resulting imaginary and real parts of the impedance are shown in Figs. 4 and 5, respectively.

The real part of the impedance can be related to the wiggler radiation spectrum $I(\omega)$ [31]:

$$
\operatorname{Re} Z(\omega)=\frac{\pi}{e^{2}} I(\omega)
$$

The spectrum $I(\omega)$ in the limit $K \gg 1$ is calculated in

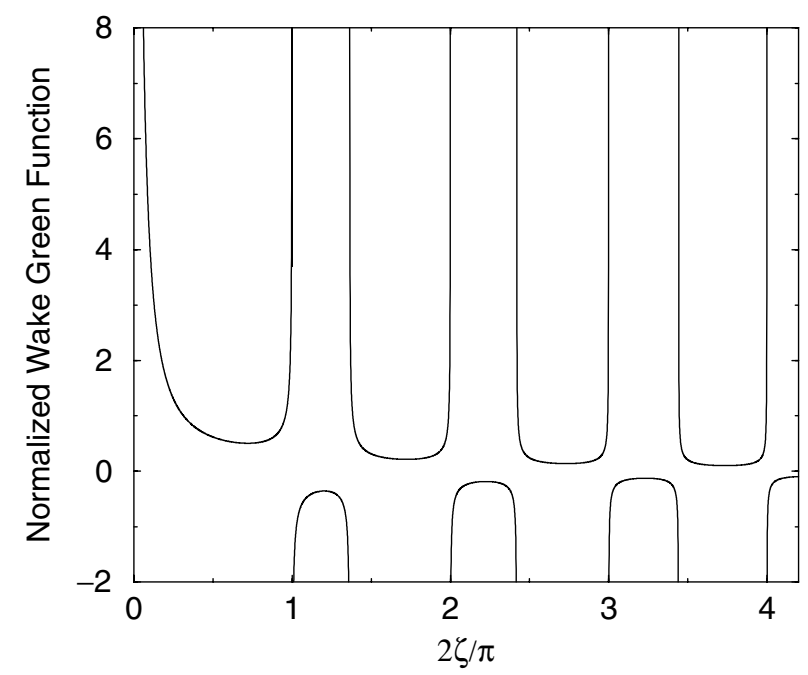

FIG. 3. The normalized wake Green function $-w(s)\left[K /\left(\gamma k_{w}\right)\right]^{2}$ as a function of the normalized coordinate $2 \zeta / \pi$.

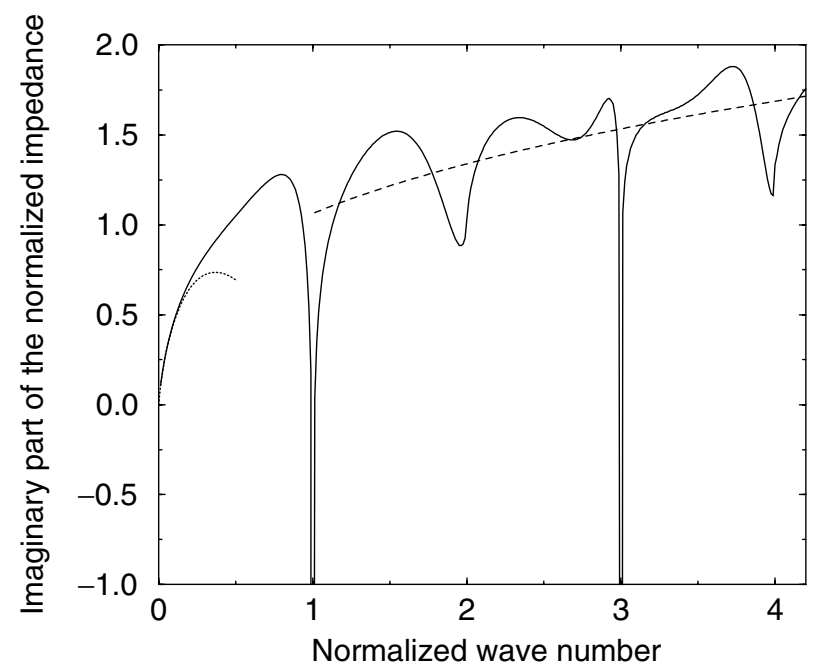

FIG. 4. The imaginary part of the normalized impedance $Z(k) / k_{w}$ as a function of the normalized wave number $k / k_{0}$. Solid line: numerical solution from Eq. (23); dotted line: analytical low-frequency asymptotic behavior from Eq. (26); and dashed line: analytical high-frequency asymptotic behavior from Eq. (27).

Appendix E. It shows a perfect agreement with the result presented in Fig. 5.

Simple analytical formulas for the impedance can be obtained in the limit of low and high frequencies.

The low-frequency impedance corresponds to the first term in Eq. (22) for function $G$ which does not oscillate with $\zeta$ :

$$
G(\zeta)=-\frac{1}{2 \zeta}
$$

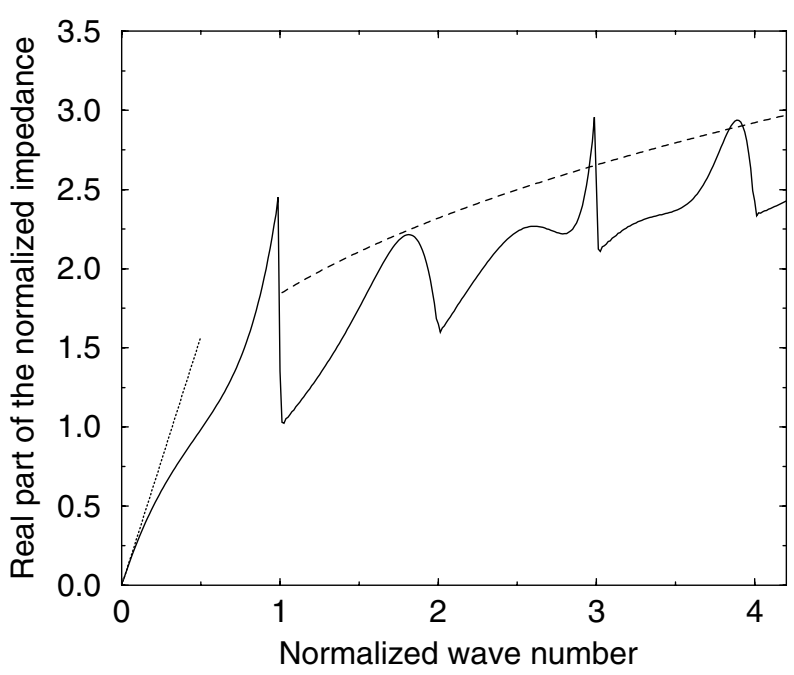

FIG. 5. The real part of the normalized impedance $Z(k) / k_{w}$ as a function of the normalized wave number $k / k_{0}$. Solid line: numerical solution from Eq. (23); dotted line: analytical lowfrequency asymptotic behavior from Eq. (26); and dashed line: analytical high-frequency asymptotic behavior from Eq. (27). 
Using the definition in Eq. (23), we then obtain the lowfrequency asymptotic behavior of the impedance as

$$
\begin{aligned}
Z(k) & =-i 2 k_{w} \frac{k}{k_{0}}\left[\gamma_{\mathrm{E}}+\log \left(\frac{4 k}{k_{0}}\right)+i \frac{\pi}{2}\right] \\
& \approx \pi k_{w} \frac{k}{k_{0}}\left[1-\frac{2 i}{\pi} \log \left(\frac{k}{k_{0}}\right)\right],
\end{aligned}
$$

where, $\gamma_{\mathrm{E}} \approx 0.5772$ is the Euler gamma constant. This asymptotic low-frequency impedance is plotted in Figs. 4 and 5 for comparison with the numerical solution.

Since we have an analytical expression for the shortrange $G(\zeta)$ in Eq. (14), we get the asymptotic highfrequency impedance as

$$
\begin{aligned}
Z(k) & =-i \frac{6 \Gamma\left[\frac{11}{6}\right]}{5 \sqrt{\pi} \Gamma\left[\frac{4}{3}\right]} A\left(\frac{K k_{w}}{\gamma}\right)^{2 / 3} k^{1 / 3} \\
& \approx-0.71 i A\left(\frac{K k_{w}}{\gamma}\right)^{2 / 3} k^{1 / 3},
\end{aligned}
$$

with $A=3^{-1 / 3} \Gamma(2 / 3)(\sqrt{3} i-1) \approx 1.63 i-0.94 \quad[18]$. This asymptotic high-frequency impedance is plotted in Figs. 4 and 5 for comparison with the numerical solution.

\section{DISCUSSION AND CONCLUSION}

In this paper, we derived the wakefield and the impedance for wigglers with $K^{2} / 2 \gg 1$ due to the synchrotron radiation. Analytical asymptotic results are obtained for the wakes in the limit of small and large distances and for the impedance in the limit of small and high frequencies. The results obtained in this paper are used for the beam instability study due to the synchrotron radiation in wigglers [34].

\section{ACKNOWLEDGMENTS}

The authors thank Dr. A. W. Chao, Dr. S. A. Heifets, and Dr. Z. Huang of Stanford Linear Accelerator Center and Dr. S. Krinsky, Dr. J. B. Murphy, and Dr. J. M. Wang of National Synchrotron Light Source, Brookhaven National Laboratory for many discussions. This work was supported by the U.S. Department of Energy under Contract No. DE-AC03-76SF00515.

\section{APPENDIX A: DETAILS FOR DERIVING EQ. (10)}

In the limit of $\Delta \ll 1$, according to Eq. (3), $B \sim \Delta^{2}$. In the numerator of the second term on the right-hand side of Eq. (2), we would have $K^{2} B(\Delta, \hat{z})[\sin \Delta \cos \hat{z}+(1-$ $\cos \Delta) \sin \hat{z}] \sim K^{2} \Delta^{3} \gg \Delta$, as long as $\Delta \gg 1 / K$. This is allowed, since we are interested in the limit of $K \gg 1$. Hence, $\Delta$ is neglected in the numerator. In the denominator of the second term, then $K^{2} B^{2} \sim K^{2} \Delta^{4} \gg \Delta^{2}$, as long as $\Delta \gg 1 / K$; hence $\Delta^{2}$ is dropped. Therefore, the second term of Eq. (2) is on the order of $1 / \Delta$. According to Eq. (4), in the limit of $\Delta \ll 1$, we have $\hat{s} \sim K^{2} \Delta$, so the first term of Eq. (2) is on the order of $1 /\left(K^{2} \Delta\right)$, and hence is much smaller than the second term in the limit of $K \gg$ 1 . Therefore the first term $1 / \hat{s}$ could be dropped. All these considerations lead us to Eq. (10).

For $\Delta \sim 1$ and $K \gg 1$, according to Eq. (4), we have $\hat{s} \sim K^{2} \gg 1$. Equation (3) suggests that $B(\Delta, \hat{z}) \sim 1$. Now, in Eq. (2), in the limit of $K \gg 1$, we can neglect $\Delta$ in comparison with $K^{2}$ in the numerator and $\Delta^{2}$ in the denominator of the second term on the right-hand side. We then note that the second term is on the order of 1 and is much larger than the first term $1 / \hat{s} \sim K^{-2} \ll 1$; hence we can drop $1 / \hat{s}$ to obtain Eq. (10).

Now let us study the limit of $\Delta \gg 1$. Equation (3) suggests that $B(\Delta, \hat{z}) \sim \Delta$. For $K \gg 1$, then in Eq. (2), $\Delta$ and $\Delta^{2}$ could be dropped in the numerator and denominator of the second term on the right-hand side, respectively. The second term is on the order of $1 / \Delta$. Now, according to Eq. (4), in the limit of $\Delta \gg 1$, we have $\hat{s} \sim$ $K^{2} \Delta$. Hence, in the limit of $K \gg 1$, the first term of Eq. (2), which is on the order of $1 /\left(K^{2} \Delta\right)$, is negligible, compared with the second term. Hence, we obtained Eq. (10).

So, in general, for large $K$, as long as $\Delta$ is not too small, i.e., $\Delta \gg 1 / K$, the simplification leading to Eq. (10) is always acceptable.

\section{APPENDIX B: SINGULAR POINTS IN $D(\zeta, \hat{z})$}

To find the scaling of the singularity, we assume that at the vicinity of the zeros $\hat{z}_{s}$ of $B(\Delta, \hat{z})$, the leading term scales as

$$
B \approx b\left(\hat{z}-\hat{z}_{s}\right)^{\alpha}
$$

then we have

$$
B^{\prime} \approx \alpha b\left(\hat{z}-\hat{z}_{s}\right)^{\alpha-1},
$$

where the prime indicates the derivative with respect to $\hat{z}$.

Let us first calculate $B^{\prime}$. From Eq. (3) we have

$$
\begin{aligned}
B^{\prime}= & -\sin \hat{z}+\sin (\hat{z}-\Delta)+\cos (\hat{z}-\Delta) \Delta \\
& -\cos (\hat{z}-\Delta) \Delta \Delta^{\prime} .
\end{aligned}
$$

To find $\Delta^{\prime}$, we revert to Eq. (9), where we find

$$
\Delta^{\prime}=\frac{C(\Delta, \hat{z})}{B^{2}(\Delta, \hat{z})}
$$

with

$$
C(\Delta, \hat{z})=2 \sin (2 \hat{z}-\Delta) \sin \left(\frac{\Delta}{2}\right) \Delta\left[2 \sin \left(\frac{\Delta}{2}\right)-\cos \left(\frac{\Delta}{2}\right) \Delta\right] .
$$

Note that $C(\Delta, \hat{z})$ is a well-defined function at the zeros of $B(\Delta, \hat{z})$.

Combining Eqs. (B1) and (B3)-(B5), we have, near the zeros $\hat{z}_{s}$, 


$$
B^{\prime}(\Delta, \hat{z})=\frac{\Xi}{b^{2}\left(\hat{z}-\hat{z}_{s}\right)^{2 \alpha}}
$$

where

$$
\begin{aligned}
\Xi & =-\cos (\hat{z}-\Delta) \Delta \times\left. C(\Delta, \hat{z})\right|_{\hat{z}=\hat{z}_{s} ; \Delta=\Delta\left(\zeta, \hat{z}_{s}\right)} \\
& =-\left.\cos (\hat{z}-\Delta) \Delta\left\{2 \sin (2 \hat{z}-\Delta) \sin \left(\frac{\Delta}{2}\right) \Delta\left[2 \sin \left(\frac{\Delta}{2}\right)-\cos \left(\frac{\Delta}{2}\right) \Delta\right]\right\}\right|_{\hat{z}=\hat{z}_{s} ; \Delta=\Delta\left(\zeta, \hat{z}_{s}\right)} .
\end{aligned}
$$

Here, $\hat{z}_{s}$ is defined as the solution of $B\left(\Delta\left(\zeta, \hat{z}_{s}\right), \hat{z}_{s}\right)=0$. Therefore, combining Eqs. (B2) and (B6), we find the scaling index $\alpha=1 / 3$ and $b=(3 \Xi)^{1 / 3}$. This means that $D(\zeta, \hat{z})$ has only an integrable singularity at $\hat{z}=\hat{z}_{s}$ with $D \propto\left(\hat{z}-\hat{z}_{s}\right)^{-1 / 3}$.

As a numeric illustration of the origin of the singularity, we plot in Fig. 6 functions $B[\Delta(\zeta, \hat{z}), \hat{z}]$ and $\Delta(\zeta, \hat{z})$ for $\zeta=1.0$.

This plot shows that at the point where $B=0$, both derivatives $B^{\prime}$ and $\Delta^{\prime}$ are infinite, in accordance with Eqs. (B2) and (B4).

\section{APPENDIX C: SIMPLE PHYSICS MODEL}

Here, we give some explanation about the peaks and the zeros in Fig. 1 based on a simple physics model.

The CSR wake is actually the field emitted by a trailing particle which acts on the particle in front. Using a model presented in Ref. [8], the longitudinal force on the leading particle can be thought of as the component of the transverse Coulomb field of a line charge projected onto the leading particle's direction of motion:

$$
W_{\|}(s, z)=e E_{\perp}(s, z) \sin \theta,
$$

where $E_{\perp}(s, z) \approx 2 N e \lambda(s, z) / r(s, z)$ is the magnitude of the transverse Coulomb electric field at the retarded position from the line charge at a retarded time. Here, $\mathrm{Ne}$ is the total bunch charge, and $\lambda$ is the function of linear

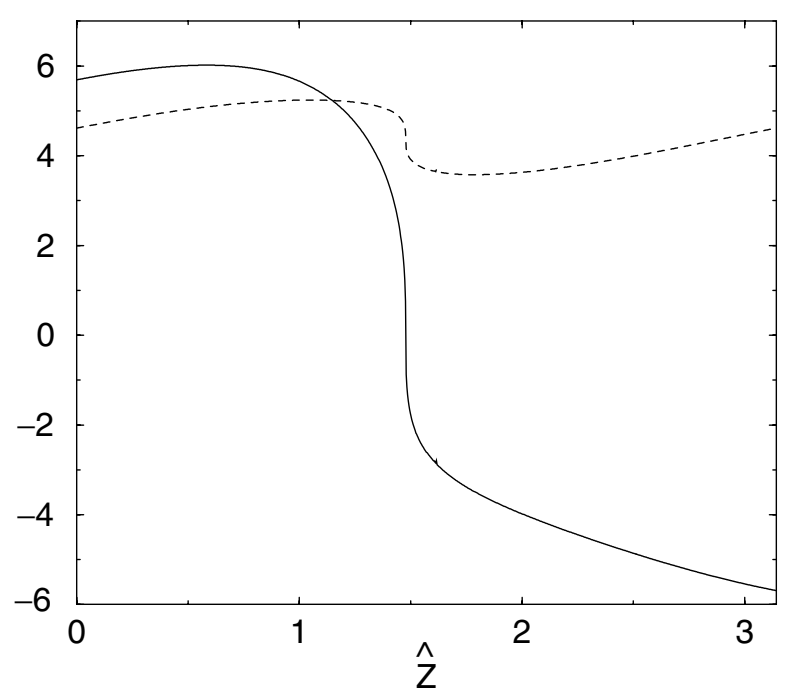

FIG. 6. A concrete example for $\zeta=1.0$. Solid line: $B(\Delta(\zeta=$ $1.0, \hat{z}), \hat{z})$ as a function of $\hat{z}$; dashed line: $\Delta(\zeta=1.0, \hat{z})$ as a function of $\hat{z}$. density normalized to 1 . The line charge is in the direction of the motion of the trailing particle at the retarded time, and $r$ is the distance of the front particle to the line charge. The argument $z$ indicates that the amplitude of the transverse electric field is actually varying along the trajectory. In Eq. (C1), $\theta$ is the angle between the direction of motion at the retarded time of the trailing particle and that of the particle at the front at the test time. To illustrate the model, we give a schematic plot in Fig. 7. In Eq. (26) of Ref. [8], it was proven that, for a uniform bunch, the energy loss gradient along the bunch is given exactly by Eq. (C1), i.e., $d \mathcal{E}(s, z) /(c d t)=W_{\|}(s, z)$. It was proven in Eq. (17) of Ref. [30] that $d \mathcal{E}(s, z) /(c d t)=$ $e^{2} k_{w} \lambda D(\hat{s}, K, \hat{z})$. Now, according to Eq. (6), one then finds, $G(s) \propto\left\langle W_{\|}(s, z)\right\rangle \equiv\left[\int_{0}^{\lambda_{w}} W_{\|}(s, z) d z\right] / \lambda_{w}$.

To understand the wiggler wakefield, let us look at the four electrons in Fig. 8. The pair with solid arrows is separated by an integer number times of the wiggler fundamental radiation wavelength. During their journey, when the light emitted by the trailing electron catches the electron in front, the instantaneous direction of motion of the front electron is always parallel to the direction of motion of the trailing electron at the retarded time when it emitted the light. Hence we have $\theta=0$. So according to Eq. (C1), the longitudinal force is always zero. It is worth noting that, at particular locations, e.g., the location in Fig. 8, the distance $r=0$, which is a singular point of $E_{\perp}$. However, since we averaged over one period, this $r=0$ point contributes only a zero measure, but $\theta=0$ for the entire period, hence $W_{\|}=0$. This explains the zeros in the longitudinal wake potential $G(s)$ plotted in Fig. 1.

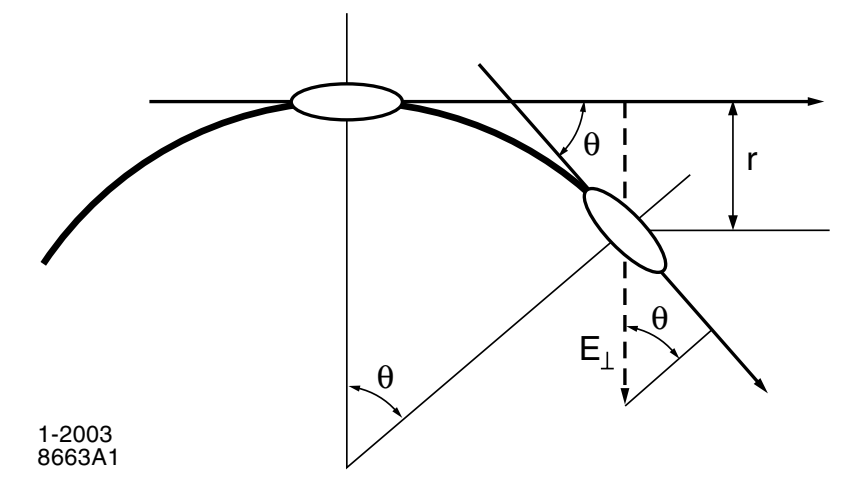

FIG. 7. Physics model to explain the longitudinal CSR wake. The two ellipses stand for a pair of electrons satisfying the retarded condition, i.e., the trailing electron is shown at the retarded time and the front electron at the test time. 


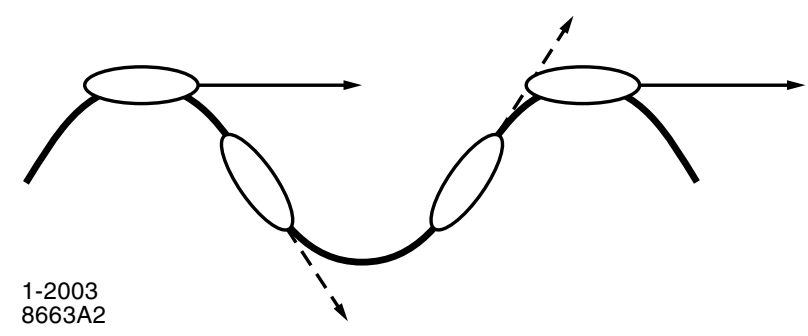

FIG. 8. Physics model to explain the peaks and zeros of $G(s)$ shown in Fig. 1. The solid line stands for the electron trajectory. The ellipses stand for the electrons. The arrows stand for the instantaneous direction of the motion. We group the four electrons into two pairs, one with solid arrows and the other with dashed arrows. Again, the trailing electron in each pair satisfying the retarded condition is shown at the retarded time, and the front electron at the test time.

The pair with dashed arrows is separated by an odd integer number times of half of the wiggler fundamental radiation wavelength. Averaged over one period, they make the largest angle between the instantaneous direction of motion of the front electron and the direction of motion of the trailing electron at the retarded time. One could check that, even though $\theta$ is changing sign during one period, $E_{\perp}$ is also changing direction. Therefore, $W_{\|}=e E_{\perp} \sin \theta$ is always pointing forward along the direction of motion of the front electron. Hence, according to Eq. $(\mathrm{C} 1), W_{\|}$reaches a maximum, and $G(s)$ also reaches a maximum. This explains the peaks shown in Fig. 1.

Let us calculate the values at these cusp points. According to Eq. (9), when $\Delta=2 n \pi$, we have $\zeta=$ $n \pi / 2$. According to Eq. (11), the numerator of the inte- grand is zero at $\Delta=2 n \pi$, while $B(\Delta, \hat{z})=2 n \pi \sin \hat{z}$ according to Eq. (3). Hence the integral is zero. So we have $G(n \pi / 2)=0$ for $n=1,2, \ldots$, . We find that it is true in the numerical solution in Fig. 1.

At $\Delta=(2 n+1) \pi$, according to Eqs. (3) and (11), we get

$$
\begin{aligned}
G(\zeta) & =\frac{2}{\pi} \int_{0}^{\pi} d \hat{z} \frac{2 \sin \hat{z}}{2 \cos \hat{z}-(2 n+1) \pi \sin \hat{z}} \\
& =-\frac{4(2 n+1) \pi}{4+[(2 n+1) \pi]^{2}} \\
\text { for } \Delta & =(2 n+1) \pi .
\end{aligned}
$$

According to Eq. (9), we have

$$
\zeta=\frac{\Delta}{4}-\frac{2}{\Delta} \cos ^{2} \hat{z} \approx \frac{\Delta}{4}-\frac{1}{\Delta} \text { for } \Delta=(2 n+1) \pi .
$$

In the above approximation, we average $\zeta$ over one period in $\hat{z}$. This becomes a good approximation, when $\Delta$ is large. This manifests itself in Fig. 1. As we find from Fig. 1, with the increasing of $\zeta$, therefore, the increasing of $\Delta$, the above approximate value gets closer and closer to the numerical solution. Combining the results at the zeros and the peaks, we get Eq. (12).

\section{APPENDIX D: CALCULATION FOR THE FOURIER COEFFICIENTS}

Since we find that the function $\bar{F}(\zeta)$ is periodic in $\zeta$ with a period of $\pi / 2$, we could represent it in a Fourier series. The calculation for the Fourier coefficients are straightforward. We here illustrate one example. For $m=$ $1,2, \ldots$,

$$
\begin{aligned}
\langle\bar{F}(\zeta) \cos [4(2 m+1) \zeta]\rangle & \equiv \frac{4}{\pi} \int_{0}^{(\pi / 2)} d \zeta \bar{F}(\zeta) \cos [4(2 m+1) \zeta] \\
& =\frac{2}{\pi^{2}} \int_{0}^{(\pi / 2)} d \zeta \cos [4(2 m+1) \zeta] \int_{0}^{\pi} d \hat{z} \frac{\sin \hat{z}}{\sin (\hat{z}-\Delta)} \\
& =\frac{1}{\pi^{2}} \int_{0}^{2 \pi} d \Delta \int_{0}^{\pi} d \hat{z} \sin \hat{z} \sin (\hat{z}-\Delta) \cos \left[(2 m+1) \Delta-\frac{(2 m+1) \sin [2(\Delta-\hat{z})]}{2}-\frac{(2 m+1) \sin 2 \hat{z}}{2}\right] .
\end{aligned}
$$

Notice that, we have used the definition of $\bar{F}$ in Eq. (20). We also changed integral variable pair $(\zeta, \hat{z})$ to $(\Delta, \hat{z})$, using the Jacobian obtained from Eq. (15). To complete the integral in Eq. (D1), we make use of the well-known identities

$$
\cos (z \cos \theta)=\sum_{n=0}^{\infty} \epsilon_{2 n}(-)^{n} J_{2 n}(z) \cos 2 n \theta,
$$

with, $\epsilon_{0}=1, \epsilon_{n}=2$ for $n=1,2, \ldots$, and

$$
\sin (z \cos \theta)=2 \sum_{n=0}^{\infty}(-)^{n} J_{2 n+1}(z) \cos (2 n+1) \theta .
$$

All the other Fourier coefficients, including the average value $\langle\bar{F}(\zeta)\rangle=-1 / 2$, are obtained in the same manner. We then obtain the Fourier series representation in Eq. (21).

\section{APPENDIX E: REAL PART OF THE IMPEDANCE AND THE WIGGLER RADIATION SPECTRUM}

Our approach to calculate $I(\omega)$ is based on the paper of Alferov et al. [32] and that of Krinsky et al. [33]. The only difference is that we are dealing with the large $K$ case; hence we could eliminate $K$ from the equations as was done for the impedance. Let us illustrate this in the following. The energy radiated per electron per unit solid 
angle per unit frequency interval per unit length is given by

$$
\frac{d I(\omega)}{d \Omega}=\frac{e^{2} \gamma^{2} N}{c \lambda_{w}} \sum_{m=1}^{\infty} G_{m}(K, \gamma \theta, \phi) H_{m}\left(\frac{\omega}{\omega_{1}}\right)
$$

where $m$ is the harmonic number of frequencies in the spectrum, and $N$ is the number of periods of the wiggler. The polar coordinates $\theta$ and $\phi$ are defined so that $\theta=0$ corresponding to the forward direction along the wiggler axis, and $\phi=0$ to be the plane of electron motion. For a large $K, \omega_{1} \approx 2 c k_{w} \gamma^{2} /\left(K^{2} / 2+\gamma^{2} \theta^{2}\right)$ is the fundamental radiation frequency, and

$$
G_{m}(K, \gamma \theta, \phi) \approx \frac{4 m^{2}}{\left(\frac{1}{2} K^{2}+\gamma^{2} \theta^{2}\right)^{2}}\left\{\left[S_{1} \gamma \theta \cos \phi-\left(S_{1}+\frac{2}{m} S_{2}\right)^{\frac{1}{2} K^{2}+\gamma^{2} \theta^{2}} \frac{2 \gamma \cos \phi}{2 \gamma}+(\gamma \theta)^{2} S_{1}^{2} \sin ^{2} \phi\right\},\right.
$$

where, we define

$$
\xi_{z} \equiv \frac{K^{2}}{4\left(\frac{K^{2}}{2}+\gamma^{2} \theta^{2}\right)}
$$

and

$$
\xi_{x} \equiv \frac{2 K \gamma \theta \cos \phi}{\frac{K^{2}}{2}+\gamma^{2} \theta^{2}}
$$

and in turn

$$
S_{1} \equiv \sum_{n=-\infty}^{\infty} J_{n}\left(m \xi_{z}\right) J_{2 n+m}\left(m \xi_{x}\right)
$$

and

$$
S_{2} \equiv \sum_{n=-\infty}^{\infty} n J_{n}\left(m \xi_{z}\right) J_{2 n+m}\left(m \xi_{x}\right)
$$

For finite, but large $N$,

$$
H_{m}\left(\frac{\omega}{\omega_{1}}\right)=\frac{\sin ^{2}\left[N \pi\left(\frac{\omega}{\omega_{1}}-m\right)\right]}{\pi^{2} N^{2}\left(\frac{\omega}{\omega_{1}}-m\right)^{2}},
$$

determines the bandwidth of the radiation. In our theory, we focus on an infinite long wiggler, i.e., we are interested in the limit of $N \rightarrow \infty$. Under such conditions, we have $H_{m} \rightarrow \omega_{1} \delta\left(\omega-m \omega_{1}\right) / N$. Now we are ready to get the radiation spectrum by integrating over the entire solid angle.

$$
I(\omega)=\int_{0}^{\pi} d \theta \sin \theta \int_{0}^{2 \pi} d \phi \frac{d I(\omega)}{d \Omega}
$$

Because of the $\delta$ function in $H_{m}$, the integral over $\theta$ could be done first. Also due to the fact that $\theta$ is small, we approximate $\sin \theta \approx \theta$. Then the integral in Eq. (E8) is reduced into a $1 \mathrm{D}$ integral as the following:

$$
I(k)=\frac{4 e^{2}}{c \lambda_{w}} \sum_{m=1}^{\infty} \int_{-(\pi / 2)}^{(\pi / 2)} d \phi\left(m-\frac{k}{k_{0}}\right)\left\{\left[S_{1} \cos \phi-\left(S_{1}+\frac{2}{m} S_{2}\right) \frac{m}{2 \cos \phi\left(m-\frac{k}{k_{0}}\right)}\right]^{2}+S_{1}^{2} \sin ^{2} \phi\right\} .
$$

Notice, the integrand has a period of $\pi$; hence we need only integrate for one period. $S_{1}$ and $S_{2}$ are further simplified as

and

$$
S_{1}=\sum_{n=-\infty}^{\infty} J_{n}\left(\frac{k}{2 k_{0}}\right) J_{2 n+m}\left[\cos \phi \sqrt{\frac{8 k}{k_{0}}\left(m-\frac{k}{k_{0}}\right)}\right]
$$

$$
S_{2}=\sum_{n=-\infty}^{\infty} n J_{n}\left(\frac{k}{2 k_{0}}\right) J_{2 n+m}\left[\cos \phi \sqrt{\frac{8 k}{k_{0}}\left(m-\frac{k}{k_{0}}\right)}\right] .
$$

Now based on Eqs. (24) and (E9), we could compute the real part of the impedance as

$$
\operatorname{Re}[Z(k)] \equiv 2 k_{w} \sum_{m=1}^{\infty} \int_{-(\pi / 2)}^{(\pi / 2)} d \phi\left(m-\frac{k}{k_{0}}\right)\left\{\left[S_{1} \cos \phi-\left(S_{1}+\frac{2}{m} S_{2}\right) \frac{m}{2 \cos \phi\left(m-\frac{k}{k_{0}}\right)}\right]^{2}+S_{1}^{2} \sin ^{2} \phi\right\}
$$

Notice $K$ is eliminated from this equation, as long as $K$ is large. We sum up the first 30 harmonics to obtain the real part of the normalized impedance up to the 4th harmonic. Adding higher harmonics will not change the impedance within this frequency region within numerical accuracy. The result is identical to Fig. 5, supporting the validity of our calculation.
[1] T. O. Raubenheimer, in Progress in the Next Linear Collider Design, AIP Conf. Proc. No. 578 (AIP, New York, 2001), pp. 53-64; NLC ZDR Design Group, SLAC Report No. 474, 1996.

[2] "TESLA: The Superconducting Electron-Positron Linear Collider with an Integrated X-Ray Laser Laboratory," Technical Design Report, DESY, 2001. 
[3] Linac Coherent Light Source (LCLS) Conceptual Design Report No. SLAC-R-593, UC-414, 2002.

[4] R. L. Warnock and P. Morton, Part. Accel. 25, 113 (1990).

[5] K.-Y. Ng, Part. Accel. 25, 153 (1990).

[6] B. E. Carlsten and T. O. Raubenheimer, Phys. Rev. E 51, 1453 (1995).

[7] J. B. Murphy, S. Krinsky, and R. L. Gluckstern, Part. Accel. 57, 9 (1997); J. B. Murphy, S. Krinsky, and R. L. Gluckstern, in Proceedings of the 1995 IEEE Particle Accelerator Conference (IEEE, Piscataway, NJ, 1996), p. 2980; A. Faltens and L. J. Laslett, Part. Accel. 4, 152 (1973).

[8] Ya. S. Derbenev et al., DESY Report No. DESY-TESLAFEL-95-05, 1995.

[9] Ya. S. Derbenev and V.D. Shiltsev, SLAC Report No. SLAC-PUB-7181, 1996.

[10] M. Dohlus and T. Limberg, Nucl. Instrum. Methods Phys. Res., Sect. A 393, 494 (1997).

[11] E. L. Saldin, E. A. Schneidmiller, and M.V. Yurkov, Nucl. Instrum. Methods Phys. Res., Sect. A 398, 373 (1997); G. Stupakov and P. Emma, in Proceedings of the 2002 European Particle Accelerator Conference, Paris, France (EPS-IGA/CERN, Geneva, 2002), p. 1479.

[12] R. Li, in Proceedings of the 1998 European Particle Accelerator Conference, Stockholm, Sweden (IOP, Bristol, U.K., 1998), p. 1230; R. Li, Nucl. Instrum. Methods Phys. Res., Sect. A 429, 310 (1999).

[13] H. Braun, F. Chautard, R. Corsini, T. O. Raubenheimer, and P. Tenenbaum, Phys. Rev. Lett. 84, 658 (2000).

[14] H. Braun, R. Corsini, L. Groening, F. Zhou, A. Kabel, T. O. Raubenheimer, R. Li, and T. Limberg, Phys. Rev. ST Accel. Beams 3, 124402 (2000).

[15] S. Heifets, G. Stupakov, and S. Krinsky, Phys. Rev. ST Accel. Beams 5, 064401 (2002).

[16] Z. Huang and K.-J. Kim, Phys. Rev. ST Accel. Beams 5, 074401 (2002).

[17] E. L. Saldin, E. A. Schneidmiller, and M. V. Yurkov, Nucl. Instrum. Methods Phys. Res., Sect. A 483, 516 (2002).

[18] G. Stupakov and S. Heifets, Phys. Rev. ST Accel. Beams 5, 054402 (2002).

[19] J. M. Byrd et al., in Proceedings of the 2002 European Particle Accelerator Conference, Paris, France (Ref. [11]), p. 659; J. M. Byrd et al., Phys. Rev. Lett. 89, 224801 (2002).
[20] ̊.. Anderson et al., Opt. Eng. 39, 3099 (2000).

[21] M. Abo-Bakr et al., in Proceedings of the 2000 European Particle Accelerator Conference, Vienna, Austria (CERN, Geneva, 2000), p. 720; M. Abo-Bakr et al., Phys. Rev. Lett. 88, 254801 (2002).

[22] U. Arp et al., Phys. Rev. ST Accel. Beams 4, 054401 (2001).

[23] G. L. Carr et al., Nucl. Instrum. Methods Phys. Res., Sect. A 463, 387 (2001); G. L. Carr et al., Nature (London) 420, 153 (2002).

[24] B. Podobedov et al., in Proceedings of the 2001 IEEE Particle Accelerator Conference, Chicago, IL (IEEE, Piscataway, NJ, 2002), p. 1921; S. Kramer et al., in Proceedings of the 2002 European Particle Accelerator Conference, Paris, France (Ref. [11]), p. 1523.

[25] J.-M. Wang, Phys. Rev. E, 58, 984 (1998); J.-M. Wang and C. Pellegrini, Brookhaven National Laboratory Report No. BNL-51236, 1979.

[26] J. B. Murphy and J. M. Wang, Nucl. Instrum. Methods Phys. Res., Sect. A 458, 605 (2001).

[27] M. Venturini and R. Warnock, Phys. Rev. Lett. 89, 224802 (2002).

[28] A. Wolski, "NLC Damping Rings: Lattice Parameters, Information and Resources," URL: http://awolski. lbl.gov/nlcdrlattice/default.htm.

[29] Y.-H. Chin, in Proceedings of the 4th Advanced ICFA Beam Dynamics Workshop on Collective Effects in Short Bunches, Tsukuba, Japan, 1990 (Lawrence Berkeley National Laboratory Report No. LBL-29981, ESG-118, 1990).

[30] E. L. Saldin, E. A. Schneidmiller, and M.V. Yurkov, Nucl. Instrum. Methods Phys. Res., Sect. A 417, 158 (1998).

[31] A.W. Chao, Physics of Collective Beam Instabilities in High Energy Accelerators (Wiley, New York, 1993).

[32] D. F. Alferov, Yu. A. Bashmakov, and E. G. Bessonov, Zh. Tekh. Fiz. 43, 2126 (1973); [Sov. Phys. Tech. Phys. 18, 1336 (1974)].

[33] S. Krinsky, M. L. Perlman, and R. E. Watson, in Handbook on Synchrotron Radiation, edited by E. E. Koch (North-Holland, Amsterdam, 1983), Vol. 1, p. 65.

[34] J. Wu, G. V. Stupakov, and T. O. Raubenheimer, SLAC Report No. SLAC-PUB-9629, 2003. 